Research Paper

\title{
Investigation of Driving Behavior and Cognitive Ability Concerning Planning Process during Driving of Elderly Drivers
}

\author{
Motoki Shino ${ }^{1)}$ Michinobu Nakanishi ${ }^{1)}$ Reo Imai ${ }^{1)}$ Hiroshi Yoshitake ${ }^{1)}$ Yoshio Fujita $^{2)}$ \\ 1) The University of Tokyo, Graduate School of Frontier Sciences \\ 5-1-5 Kashiwanoha, Kashiwa, Chiba, 277-8563, Japan (E-mail:motoki@k.u-tokyo.ac.jp) \\ 2) Chiba Prefectural University of Health Sciences \\ 2-10-1 Wakaba, Mihama, Chiba, Chiba, 261-0014, Japan
}

Received on October 12, 2017

\begin{abstract}
Our research aims to investigate the relation between the driving behavior in the actual environment and the cognitive ability for elderly drivers. To examine the planning process during driving, we analyzed the driving behavior of passing through an intersection with a stop sign and lane changing during driving. As a result of the analysis, about $70 \%$ of elderly drivers didn't drive safely just like driving instructors. Moreover, it was found that elderly drivers of Mild Cognitive Impairment (MCI) group had lower divided attention and alternating attention than that of non-MCI group. The drivers of MCI group were more difficult to decide a task during driving than that of non-MCI group.
\end{abstract}

KEY WORDS: human engineering, elderly driver, driving behavior in the actual environment, cognitive ability, planning process [C2]

\section{Introduction}

In Japan, the number of fatal traffic accidents involving elderly drivers has increased to about $2,138^{(1)}$, while the total number of fatal traffic accidents has decreased. In order to decrease the total number of traffic accidents, studies on active safety technologies including driver assistance systems and driver education systems for elderly drivers are important. However, the difference of elderly drivers' cognitive ability and driving characteristics is large among individuals. Thus, it is difficult to understand the characteristics of various types of drivers. In addition, little is known about the correlation between the cognitive ability and unsafe driving behavior in the actual environment. To compensate for the decline of the cognitive ability, we need to identify the relationship between cognitive ability characteristics and unsafe driving behavior. Thus, this study aims to investigate the relationship between the driving behavior in the actual environment and the cognitive ability such as divided attention, alternating attention and inhibitory function.

\section{Overview of this research}

In this research, unsafe driving behavior is defined as the driving behavior that has a possibility of leading to traffic accidents Suzuki reported that the characteristics that caused traffic accidents and violation are physical characteristics, mental one, driving one and social one ${ }^{(2)}$. In these elderly drivers' characteristics, the decline of the cognitive ability with aging is remarkable and affects driving characteristics. Driving is the repetition of cognition, judgement and operation. In this process, cognition and judgement are the process in a driver brain, thus the decline of the cognitive ability with aging definitely affect cognition and judgement. The cognitive ability declining with age are divided attention ${ }^{(3)}$, selective attention ${ }^{(4)}$, alternating attention, inhibitory function ${ }^{(5)}$, and useful field of view (UFOV) ${ }^{(6)}$ etc. Owsley analyzed the relationship between the cognitive ability and the history in the past 5 years of elderly drivers. He concluded that older drivers with visual sensory impairment, cognitive impairment, and/or narrow useful field of view were at greater risk for crashes than those without these problems ${ }^{(6)}$. Tamida et al. reported that passing through an intersection with a stop sign and lane changing during driving were two behavior evaluated as unsafe driving behavior by driving instructors (7). However, little is known about the relationship between the cognitive ability and unsafe driving behavior in the actual environment of elderly drivers. This research focuses on elderly drivers older than 75 years. The aim of this research is to find out the relationship between their driving behavior in the actual environment and their cognitive ability such as divided attention, alternating attention and inhibitory function. The driving behavior of passing through an intersection with a stop sign and lane changing during driving were analyzed.

First, the methods of the experiments of getting the cognitive ability and the driving behavior were described. Next, the results of elderly drivers' cognitive ability test, the driving behavior and the relationship between the cognitive ability characteristics and the driving behavior characteristics were shown. In particular, the participants were classified into Mild Cognitive Impairment (MCI) group and non-MCI group based on the score of Wechslor Memory Scale-Revised (WMS-R) Logical memory test II ${ }^{(8)}{ }^{(9)}$. Then, the cognitive ability and the driving behavior were compared between the two groups to investigate the impact on the driving behavior due to the decline of the cognitive ability.

\section{Methods for the experiment}

This section describes participants, the way of recording the driving behavior with real vehicle in the actual behavior, the way 


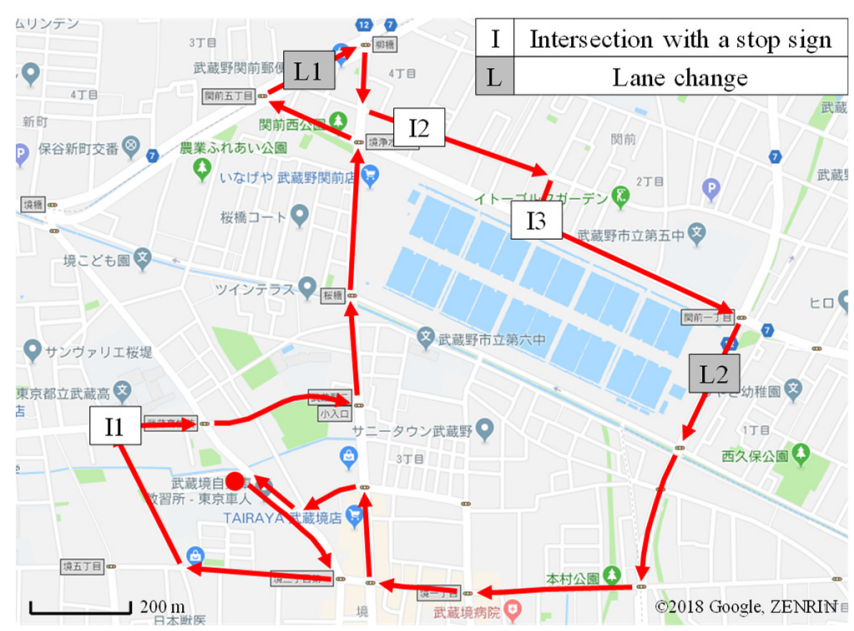

Fig. 1 Driving course ${ }^{(10)}$ used in the experiment.

of analyzing their driving behavior characteristics and the cognitive ability tests. This research was done under the approval of the ethics committee of The University of Tokyo.

\subsection{Participants}

The participants were 28 elderly males and 3 elderly females who drove routinely, and 2 driving instructors. Elderly participants was older than 75 (average age $77.6, \mathrm{SD}=2.27$ ). Every participant had general good health, drove more than once a week, and possessed a driver's license at that time.

\subsection{Driving record with real vehicle}

To examine the driving characteristics of the elderly, we recorded their natural driving behavior for about 30 minutes in the prescribed actual driving scenarios, using a drive recorder. The drive recorder device (DR, Fine fit Design, Inc.) was installed in the vehicle. To record the vehicle state and the driver operator, the DR consisted of four cameras and a three-axis acceleration sensor. One camera recorded the traffic environment and three cameras recorded the driver behavior. In addition, the signal of vehicle velocity, blinker and brake pedal operation were recorded. Fig.1 shows the driving course in the actual environment. In this driving course, the participant passed three intersections with a stop sign (Scene I1 I3) and changed lane twice (Scene L1, L2). The car used in this experiment was an instruction car. For guaranteeing the safety of the experiment, the instructor rode on the passenger seat with an auxiliary brake to stop the car in case of emergency.

\subsection{The way of classifying the driving behavior}

Passing through an intersection with a stop sign and lane changing during driving are the driving behavior that a driver needs to make a plan during driving. We focused on the planning process during driving of these driving behavior and classified the driving behavior into the stages of the planning process during driving. To define the stages of the planning process during driving, the planning process during driving considering the cognitive ability was constructed based on the previous research ${ }^{(11)(12)}$. Fig. 2 shows this planning process during driving. Driving is the repetition of cognition, judgement and operation. For safe driving, in the cognition process, a driver perceives information. In the judgement

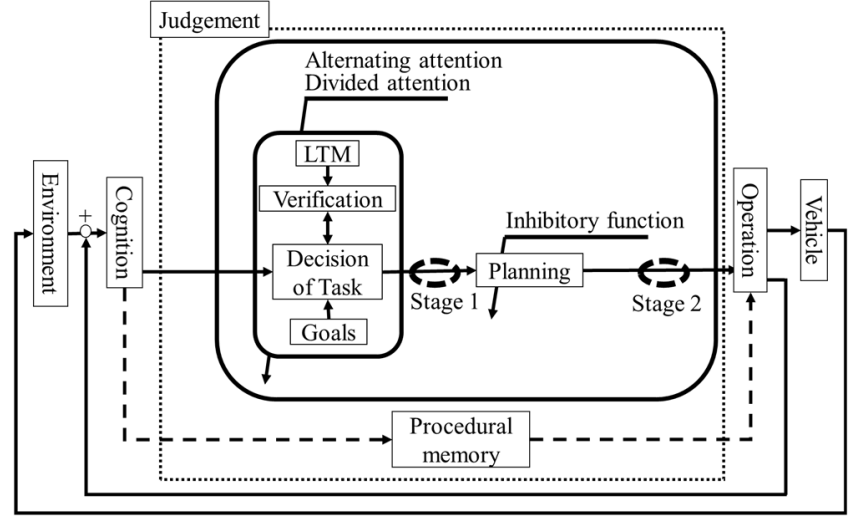

Fig. 2 Planning process during driving considering the cognitive ability.

process, in the difficult environment to operate, a driver verifies the environment and the own vehicle state by recalling long-term memory (LTM). Then, to achieve goals, a driver decides a task and plans the concrete driving behavior during driving. On the other hand, in the easy driving environment to operate, a driver judges operation unconsciously with procedural memory. For example, "When a driver is waiting for an intersection signal unconsciously, he/she steps on the gas pedal because it has changed to a green light", "When a driver is following the preceding car, he/she steps on the brake unconsciously because its tail lamp goes on" are the judgement process during driving with procedural memory. Such an unconscious driving behavior is affected by drivers' habit. In the operation process, a driver operates a vehicle and confirm according to the plan. Especially, in the judgement process, divided attention is needed to decide a task during driving because a driver needs to verify of the environment and recall LTM simultaneously. In addition, alternating attention is needed to decide a task during driving because a driver needs to switch verification and decision of task. In addition, inhibitory function is needed to make an adequate plan during driving because a driver needs to exclude plans unnecessary to complete the task. We hypothesized that elderly drivers whose cognitive ability have decreased with age were difficult to make a plan during driving consciously. Thus, we focused on the planning process during driving without procedural memory. The following approach was employed to classify the driving behavior of the stages of the planning process during driving. Judgement process during driving is difficult to be evaluated directly. Thus, we assessed his/her judgement process from driving operation indirectly. We classified the driving behavior into two stages of the planning process during driving. As shown in Fig. 2, the stage from "Decision of Task" to "Planning" was defined as Stage 1 by the appropriateness of the driver's decision of task in the near future. Moreover, the stage from "Planning" to "Operation" was defined as Stage 2 by the appropriateness of the planning during driving to complete the task. The inadequate driving behavior type in Stage 1 was "No intension to perform task" (Type 1). The inadequate driving behavior type in Stage 2 was "The lack of the behavior element" (Type 2) and "The inversion of the behavior orders" (Type 3). When a driver was able to drive just like the instructors, a driver behavior type was regarded as "Safety" (Type 4). The evaluation criteria for the 
Table 1 Classification of the driving behavior with real vehicle

\begin{tabular}{|c|c|c|c|c|}
\hline & $\begin{array}{c}\text { Driving } \\
\text { behavior type }\end{array}$ & $\begin{array}{c}\text { Driving behavior } \\
\text { characteristics }\end{array}$ & $\begin{array}{c}\text { Driving behavior at an intersection } \\
\text { with a stop sign (Scene I2) }\end{array}$ & $\begin{array}{c}\text { Driving behavior at lane changing } \\
\text { (Scene L1) }\end{array}$ \\
\hline Safe & Type 4 & Safety & Similar to the driving instructors' safe driving behavior \\
\hline \multirow{3}{*}{ Unsafe } & Type 1 & $\begin{array}{c}\text { No Intention to } \\
\text { perform task }\end{array}$ & No intention to slow down & No intention to change the lane \\
\cline { 2 - 5 } & Type 2 & $\begin{array}{c}\text { Lack of Behavior } \\
\text { elements }\end{array}$ & $\begin{array}{c}\text { The lack of the right or left } \\
\text { confirmation }\end{array}$ & $\begin{array}{c}\text { The lack of turning on the blinker or } \\
\text { the confirmation at the approaching } \\
\text { lane side }\end{array}$ \\
\hline Type 3 & $\begin{array}{c}\text { Inversion of } \\
\text { Behavior orders }\end{array}$ & $\begin{array}{c}\text { No confirmation at the blind side after } \\
\text { passing the blind disappearance points }\end{array}$ & $\begin{array}{c}\text { Turning on the blinker or the } \\
\text { confirmation at the approaching lane } \\
\text { side after steering }\end{array}$ \\
\hline
\end{tabular}

driving behavior were defined from safe driving behavior of instructors. In Table 1, the concrete evaluation criteria of passing through the intersection with a stop sign and lane changing during driving are shown. When a driver passes the intersection with a stop sign, a driver needs to slow down and then stop at the stop line. If there are blind disappearance points at the intersection, a driver needs to confirm after passing the blind disappearance points. Type 1, Type 2 ,Type 3 and Type 4 were defined as "No intention to slow down", "The lack of right or left confirmation", "No confirmation at the blind side after passing blind disappearance point" and "Similar to the driving instructors' safe driving behavior" , respectively as shown in Table 1 . When a driver changes lane during driving, a driver needs to turn on blinker, confirm at the approaching lane and steer to the approaching lane. Type 1, Type 2, Type 3 and Type 4 were defined as "No intention to change lane", "The lack of turning on blinker or confirmation at approaching lane side", "Turning on blinker or confirmation at approaching lane side after steering" and "Similar to the driving instructors' safe driving behavior", respectively as shown in Table 1 .

\subsection{Cognitive ability tests}

The following cognitive ability tests were conducted to evaluate participants' cognitive ability that affected the planning process during driving .First, to exclude individuals with dementia and to classify Mild Cognitive Impairment (MCI) group and nonMCI group, we conducted two tests. They were a mini-mental state examination (MMSE) ${ }^{(13)}$ and Wechslor Memory Scale-Revised (WMS-R) Logical memory test I and II ${ }^{(8)}{ }^{(9)}$. Next, to evaluate cognitive ability that affects the planning process during driving, we conducted two cognitive ability tests. They were visual field with inhibitory tasks (VFIT) ${ }^{(14)}$ and Trail Making Test (TMT) ${ }^{(15)}$. Divided attention and inhibitory function was evaluated based on VFIT, and alternating attention was evaluated based on TMT.

\subsubsection{Evaluation criteria to classify $\mathrm{MCI}$ and Dementia among the} participants

In Alzheimer's Disease Neuroimaging Initiative (ADNI) project, based on the scores of MMSE and Logical Memory II test, cognitive ability characteristics was classified into cognitive normal $(\mathrm{CN})$, early Mild Cognitive Impairment (EMCI), late MCI (LMCI) and Alzheimer Dementia (AD) ${ }^{(16)}$. The participants' schooling years were needed to classify the cognitive ability characteristics. In this research, their schooling years were defined as from 8 to 15 . The cutoffs of MMSE and W-MSR Logical Memory II test were defined as 23 of 30 and 8 of 50, respectively.

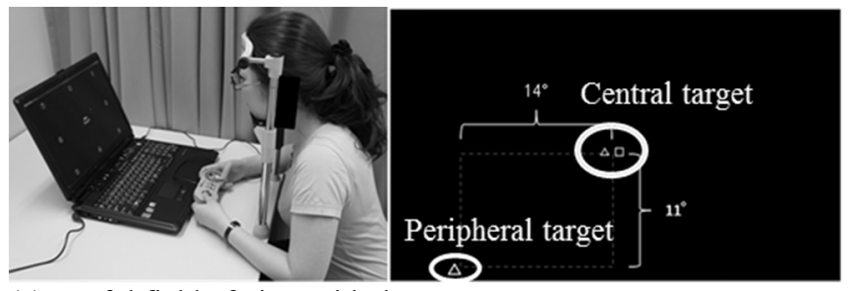

(a) Useful field of view with the VFIT-EV test.

(b) Screen display on the VFIT-EV, dual-task test (actual test). Fig. 3 Useful field of view with the VFIT-EV test.

Participants were classified into the three groups of non-MCI, MCI and AD based on the scores of MMSE and WMS-R Logical Memory II.

\subsubsection{Visual Field with Inhibitory Tasks (VFIT test) ${ }^{(14)}$}

The conventional UFOV test consists of dual-task testing with simultaneous central and peripheral targets. The test requires touch panel screen or other complicated device and is subject to bodily and language function. However, several modified methods to improve this weakness have been reported recently. Fujita et al. developed a new UFOV test, i.e., the visual field with inhibitory tasks (VFIT) to resolve these problems ${ }^{(14)}$. We used its version for screening elderly individuals (VFIT-EV). The VFIT-EV was conducted as follows. First, the examiner displayed a cross in the center of the screen to confirm the central fixation point for the participant as shown in Fig. 3. After presentation of the central fixation cross for $500 \mathrm{~ms}$, four central targets and one peripheral target were simultaneously presented for $200 \mathrm{~ms}$. The peripheral targets were 11 degrees of the visual angle positioned at the top and bottom, at 14 degrees of the visual angle positioned at the right and left, and at the square root degree of the sum of the squares of 11 and 14 at the top right, bottom right, bottom left, and top left, respectively. The participants were instructed to push a button on the right side of the controller when the two central targets differed and not to push the button when the two target were the same. After pushing a button, the figure was presented in the center of the monitor, and the participant was instructed select the same figure as presented in the periphery using a directional key on the controller. The response was recorded as correct only when the response for both the central figures and the peripheral target were correct. The correct answer rate was defined by the rate of the correct answers for 32 trials in which the center figures differed in a run. The false alarm rate was defined as the rate of wrong responses for the central target, i.e., pushing the button when the two central targets were the same. There were four stages with diffent difficulty. As the stage went up, the peripheral targets were 
presented farther from the center of the monitor.

In this research, inhibitory function was evaluated based on the average of the central error rate of dual-task test from stage 2 to stage 4 (VFIT CER). And divided attention was evaluated based on the average of the peripheral error rate of dual-task test from stage 2 to stage 4 (VFIT PER).

\section{Elderly drivers' characteristics of the cognitive ability and the driving behavior}

\subsection{Cognitive ability tests}

The results of 27 elderly participants with no loss of cognitive ability tests results were discussed.

\subsubsection{Classification Dementia and MCI among the participants}

The range of MMSE scores of 27 participants were 25-30 of 30 (cutoff 23/24). WMS-R scores of twenty-seven elderly participants are shown in Fig. 4. The range of WMS-R scores were 2-27 of 50 (cutoff 8/9). There were 6 people in MCI group and 21 people in non-MCI group. It was found that there were no participants who could have developed dementia. And, the participants were classified into the two groups of MCI and non-MCI.

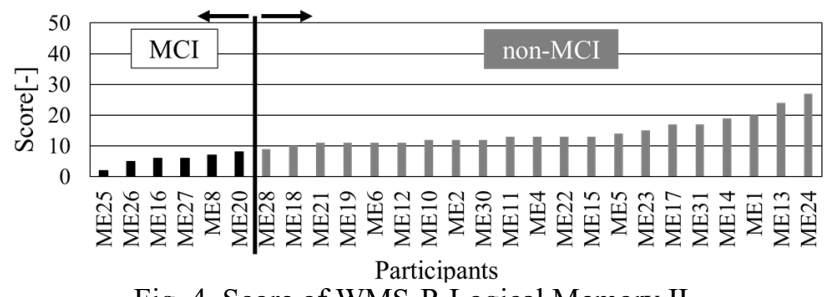

Fig. 4 Score of WMS-R Logical Memory II.

4.1.2. Comparison of the cognitive ability tests (VFIT and TMT) between MCI group and non-MCI group

Fig. 5 shows the comparison of the normalized ages and scores of the cognition ability tests between MCI and non-MCI group. We normalized the scores of the following scores (Age: 100, VFIT CER:0.1, VFIT PER:0.5 ,TMT-A: 200, TMT-B: 400, $\Delta$ TMT: 200). The significant difference was calculated by one-sided test of Mann-Whitney U test. The average age of MCI group was older than that of non-MCI group. About VFIT CER, the average score of MCI group was better than that of non-MCI group. About VFIT PER, TMT Part A, B, $\triangle \mathrm{TMT}$, the average scores of MCI group were worse that of non-MCI group. As for the scores of TMT Part $\mathrm{A}$ and $\mathrm{B}$, there was the significant difference between two groups $(\mathrm{p}<0.05)$. This shows that divided attention and alternating attention of MCI group were worse than non-MCI group and inhibitory function of MCI group was not worse than non-MCI group.

4.1.3 Comparison between divided attention, alternating attention and inhibitory function

To compare MCI group with non-MCI group about divided attention, alternating attention and inhibitory function, we investigated the result of the comparison between VFIT and TMT. As a result, there was a tendency between the scores of VFIT PER and that of TMT Part A, Part B and $\triangle T$ TMT. On the other hand, there

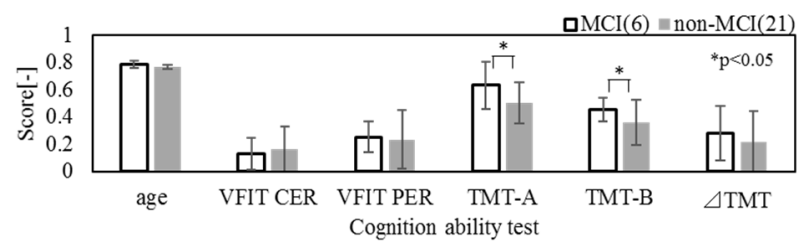

Fig. 5 The comparison of the normalized age and the normalized scores of the cognition ability tests between MCI group and non-MCI group.

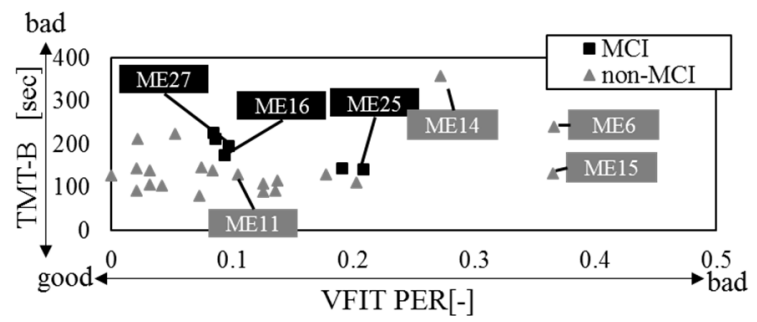

Fig. 6 VFIT PER versus TMT-B.

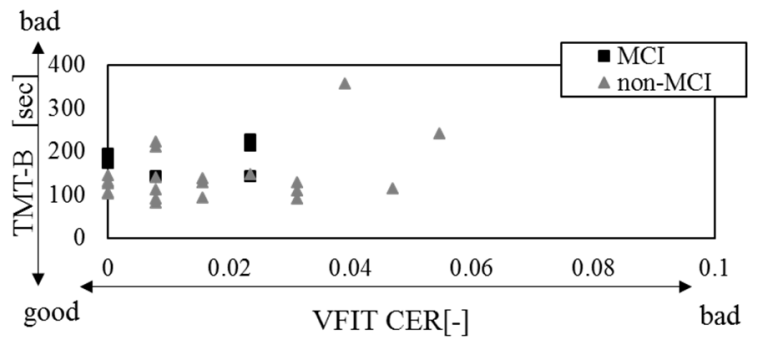

Fig. 7 VFIT CER versus TMT-B

was not a tendency between the scores of VFIT CER and that of TMT Part A, Part B and $\triangle$ TMT. Fig. 6 and Fig. 7 show the relationship between the scores of VFIT PER and the TMT Part B, the relationship of the score of VFIT CER and TMT Part B as the represented results, respectively. As shown in Fig.6, VFIT PER and TMT Part B of MCI group were worse than that of non-MCI group, apart from three participants in non-MCI group (ME6, 14 and 15). We interpreted this result of three elders as the presence of elders with low attention ability who didn't belong to MCI which was defined from medical evaluation index WMS-R. And we thought that they got lower score of VFIT PER and TMT part B due to the degradation of attention ability. Then, as shown in Fig. 7, VFIT CER and TMT Part B were not affected by whether participants belong to MCI group or not.

\subsection{Driving behavior analysis}

This research focused on the driving behavior at Scene I2 and Scene L1 at which elderly drivers' planning process during driving can be investigated. The results of 18 elderly participants with no loss of the cognitive ability tests and driving data at Scene I2 and Scene L1 were investigated.

4.2.1 Passing through an intersection with a stop sign in the actual environment (Scene I2)

Scene I2 is an intersection with a stop sign with left and right blind disappearance points as shown in Fig.8. There is a short distance between these disappearance points. In this intersection with a stop sign, the distance of the blind right and left disappearance point from the stop line are 1.5 meters and 2 meters. 


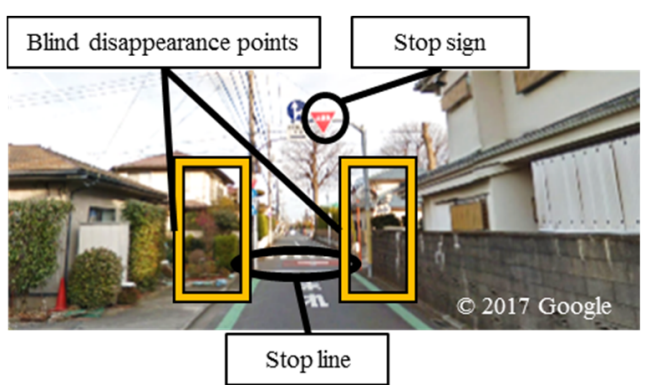

a) The road environment from the view of the driver at Scene I2.

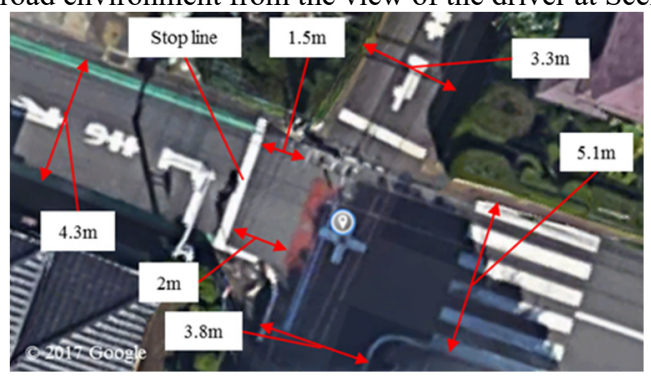

b) The road environment from the above at Scene I2.

Fig. 8 The road environment at Scene I2.

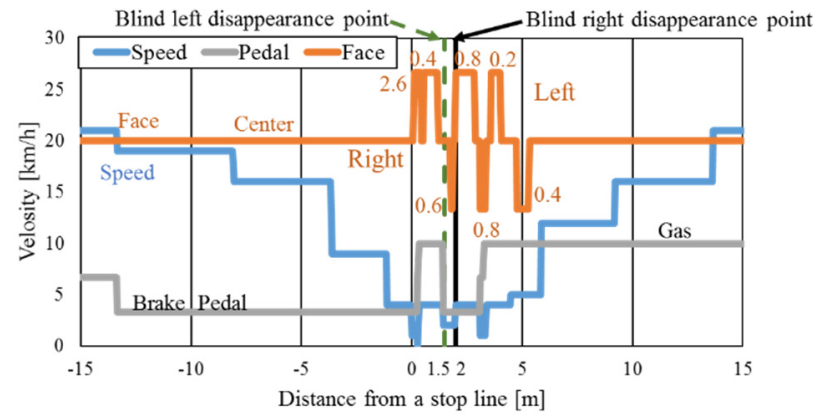

Fig. 9 Driving behavior of the instructor P2 at Scene I2.

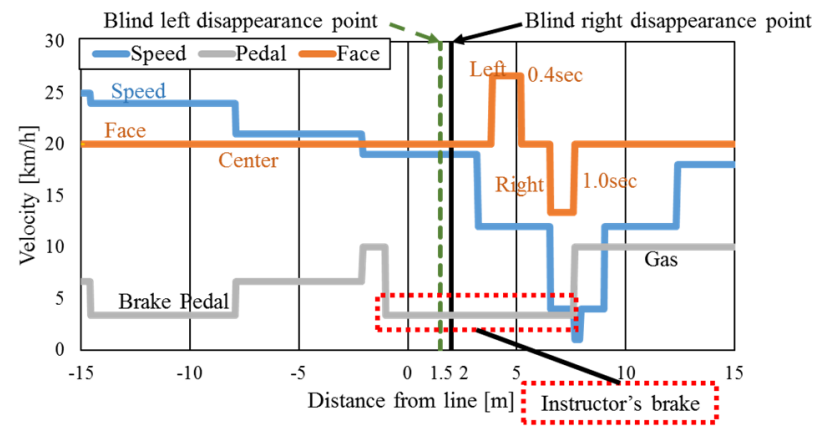

Fig. 10 Driving behavior of the MCI elderly driver ME27 at Scene I2.

The participant was asked to go straight at Scene I2. Thus, the participant needed to notice a stop sign and decide the task of stopping. Fig. 9 and Fig. 10 show the driving behavior of the instructor P2 and a represented elderly driver ME27 in MCI group, respectively. These figures' horizontal axis represents the distance from the stop line. In these figures, the blue line, the gray one and the orange one stand for velocity, pedal operation and face direction, respectively. The time of confirmation when passing through the intersection is also written near an orange line. P2 slowed down to $0 \mathrm{~km} / \mathrm{h}$ near the stop line. Next, P2 kept the low speed less than $5 \mathrm{~km} / \mathrm{h}$ when passing left and right disappearance blind points. Then, P2 confirmed right and left before and after blind disappearance blind points more than 0.6 second. This careful driving behavior expressed watching out for pedestrians, bicycles and vehicles rushing out from the crossing lane. On the other hand, ME27 did not intend to stop at the intersection with a stop sign, and then the instructor on the passenger seat stepped on auxiliary brake for guaranteeing the safety. ME27 did not stop at stop line and confirm right and left. This driving behavior was unsafe because there was a high possibility that ME27 collide pedestrians, bicycles and vehicles that rushed from the crossing lane.

4.2.2 The analysis of lane changing during driving in the actual environment (Scene L1)

At Scene L1, the participant was asked to turn right at the next intersection when the own vehicle was on the first lane as shown Fig. 11. The participant needed to decide the task of lane changing during driving. We assumed that the driving behavior of lane changing was influenced by the disturbance such as parked cars onthe first lane and vehicles on the second lane behind the own vehicle. Thus, the data of the driving behavior at Scene L1 with disturbance were excluded from analysis. Fig. 12 and Fig. 13 show the driving behavior of the instructor P1 and a represented elderly driver ME16 in MCI group, respectively. These figures' horizontal axis represents the time from the time at which the participant was asked to turn right at the next intersection. In these figures, the blue line, the gray one, the orange one, the green one and the yellow one

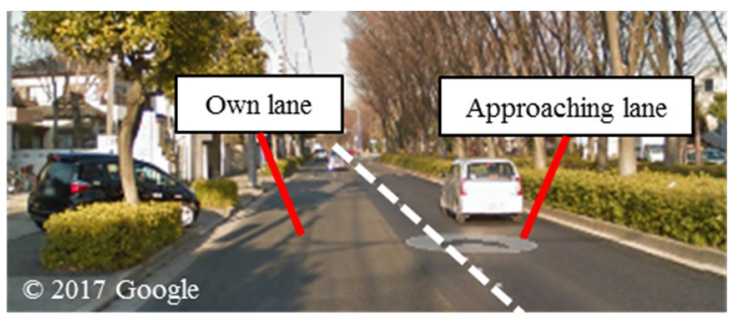

Fig. 11 The road environment of Scene L1

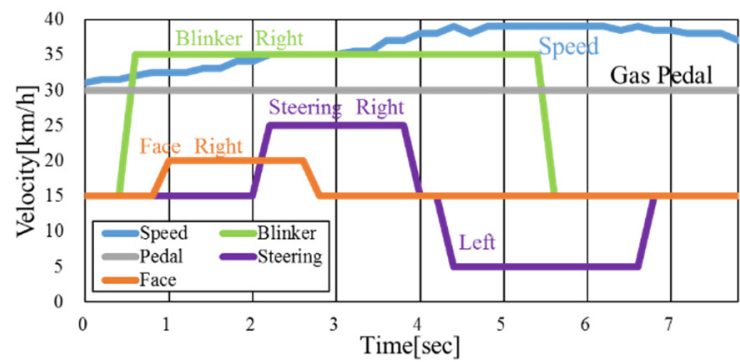

Fig. 12 Driving behavior of the instructor P1 at Scene L1.

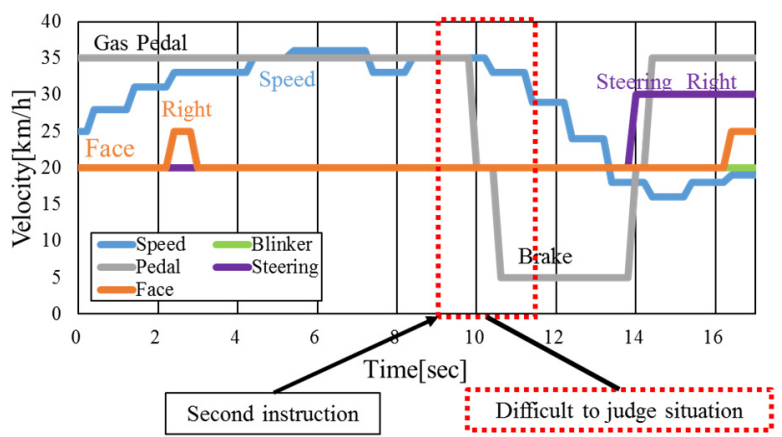

Fig. 13 Driving behavior of the MCI elderly driver ME16 at Scene L1. 
stand for velocity, pedal operation, face direction, the direction of the blinker and the direction of the steering, respectively. P1 turned on a right blinker, confirmed the right side on the first lane, operated steering to the right side and changed lane to the second lane. This careful driving behavior expressed the communication with the cars behind the own vehicle and watching out for the cars on the second line behind the own vehicle. On the other hand, ME16 did not intend to change lane with the first instruction, and then got the second instructions from the instructor on the passenger seat. When ME16 got the second instruction, he looked puzzled by the verification of the situation and stepped on brake. After understanding the next behavior, ME16 operated steering to the right side and changing lane to the second lane without right confirmation and turning on the right blinker signal. This driving behavior was unsafe because there was a high possibility that ME16 collide vehicles on the second lane.

\subsubsection{Driving behavior type of elderly drivers}

Fig. 14 shows the number of the participants of each driving behavior type. In 18 elderly drivers, the number of Type 4 as "Safety" at Scene I2 and L1 was 4 and 6, respectively. Also, the number of Type 1 as "No intention to perform task" at Scene I2 and L1 was 2 and 3, that of Type 2 as "The lack of the behavior element" was 1 and 2 and that of Type 3 as "The inversion of the behavior orders" was 11 and 7, respectively. The result indicates that about $70 \%$ of elderly drivers did not drive safely just like the instructors.

\subsection{Feature of the cognition ability and the driving behavior}

To investigate the impact on driving behavior due to the decline of the cognitive ability, the component ratio of the driving behavior type of MCI group and non-MCI group are shown in Fig. 15 and Fig.16. At both of Scene I2 and Scene L1, the component ratio of Type 1 of MCI group are larger than that of non-MCI group. As a result, it was found that MCI group is more difficult to decide a task during driving and more unsafe than non-MCI group. Four (ME11, ME16, ME25 and ME27) of 18 elderly drivers belonged to Type 1 at Scene I2 or Scene L1. Three (ME16, ME25 and ME27) of four belonged to MCI group and one (ME11) of four belonged to non-MCI group. To analyze their cognitive ability, their represented cognitive ability test scores of VFIT PER and TMT Part B are shown in Fig.6. The scores of ME16, ME25 and ME27 were worse than that of non-MCI group. And the scores of ME11 in non-MCI group was bad like that of MCI group. From the mentioned result, it was found that four elderly drivers of Type 1 had low divided attention and alternating attention.

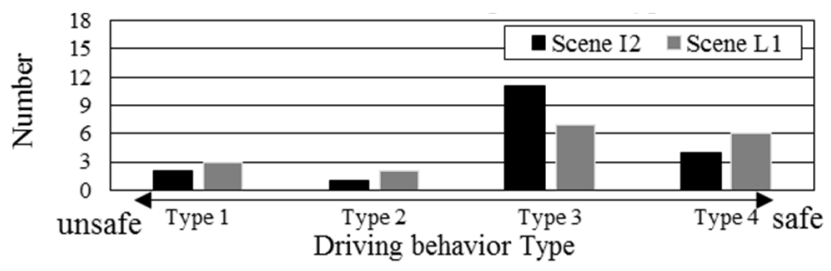

Fig. 14 The number of the driving behavior at Scene I2 and L1.

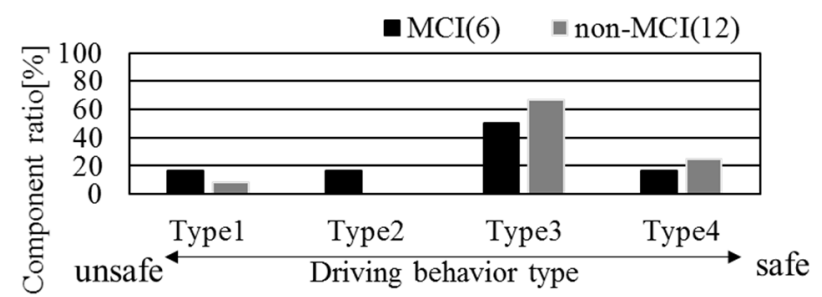

Fig. 15 The comparison of the ratio of the driving behavior type between MCI group and non-MCI group at Scene I2.

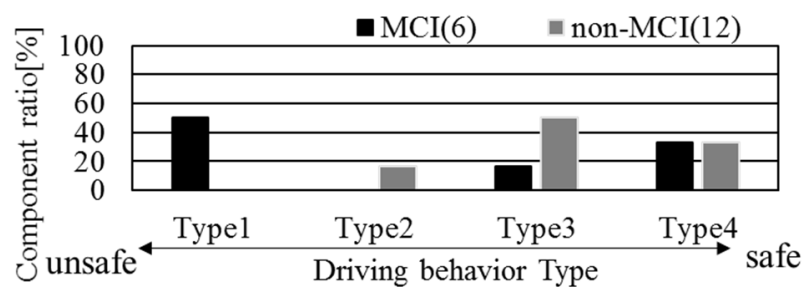

Fig. 16 The comparison of the ratio of the driving behavior type between MCI group and non-MCI group at Scene L1.

\section{Consideration on the planning process during driving}

We consider about the planning process during driving of the instructors and the elderly drivers whose driving type were Type 1 as shown in Fig. 17, Fig.18 respectively. The instructors have high level of LTM, divided attention and alternating attention. Thus, in the planning process during driving, they could verify the environment by recalling their LTM, decide a task and make a plan smoothly shown in Fig.17. On the other hand, elderly drivers of Type 1 had low LTM, divided attention and alternating attention. They could not recall LTM well by the decline of LTM, divided attention and alternating attention and they were difficult to decide a task during driving as shown in Fig.18. Then they continued to drive unconsciously with procedural memory. Thus, the elderly drivers whose driving type was "No intention to perform task" may have difficulty recalling long-term memory (LTM) due to the degradation of their cognitive ability.

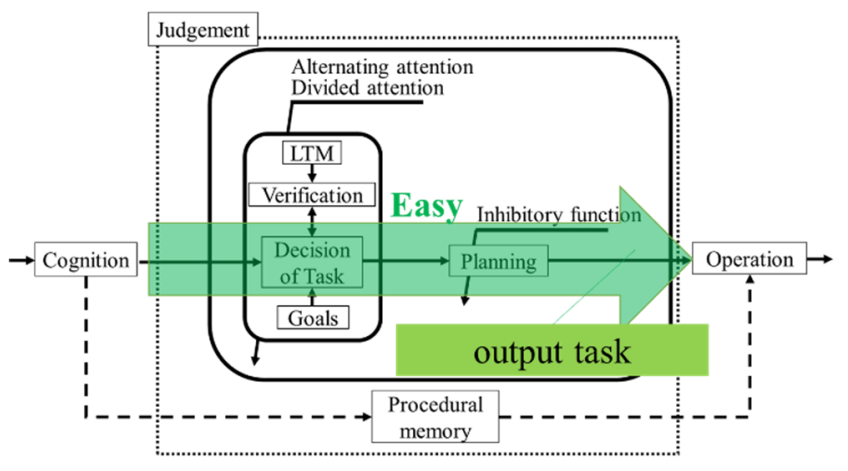

Fig. 17 The planning process during driving of the instructor. 


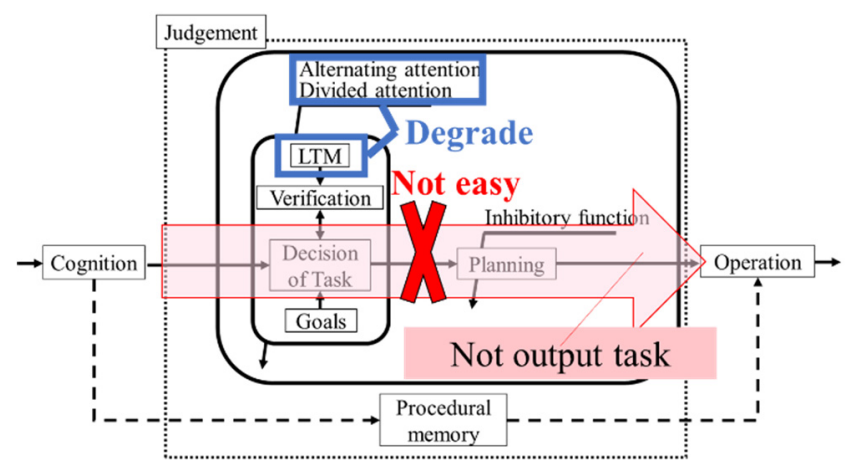

Fig. 18 The planning process during driving of elderly drivers who belonged to Type 1 .

\section{Conclusion}

This research investigated the relationship between cognitive ability and the driving behavior in the actual environment of elderly drivers. We hypothesized that elderly drivers whose cognitive ability had decreased were difficult to make a plan during driving consciously. We focused on Mild Cognitive Impairment (MCI) group and non-MCI group as research objects. The following are the conclusions of this research.

(1) Divided attention and alternating attention of MCI group were worse than non-MCI group. Inhibitory function of MCI group was not worse than non-MCI group.

(2) About $70 \%$ of elderly drivers in both of MCI group and non$\mathrm{MCI}$ group did not drive safely just like the driving instructors.

(3) Elderly drivers of MCI group were more difficult to decide a task during driving than that of non-MCI group at the intersection with a stop sign and lane changing during driving. These findings in this research will help to develop the driving education assistance system and driving assistance system to defend elderly drivers' unsafe behavior.

This paper is written based on a proceeding presented at JSAE FAST-zero'17 Meeting.

\section{Acknowledgements}

To advance our own research, we would like thank to the elderly participants and Musashisakai Driving School in Musashino City who warmly agreed to work with us. This study has done with financial assistance from Takata Foundation.

\section{References}

(1) National Police Agency: Accident statics (2017).

(2) Suzuki H.: Driving ability of elderly people, Japanese journal of clinical psychiatry, Vol. 29, No. 6, pp. 609-615 (2000).

(3) Ponds.M, Brouwer.W, Wolffelaar.P: Age Differences in Divided Attention in a Simulated Driving Task, J Gerontol, Vol. 43, No. 6, pp. 151-156 (1988).

(4) Kaneko R., Fukunaga Y., Kumada T.: Aged-Related Changes in Interface Effects of Peripheral Stimulus, VISION, Vol. 15, No. 4, pp. 245-254 (2003).

(5) Hasher L., Zacks R.: Working memory, comprehension, and aging: Areviewand a new review, In G. Bower(Ed), The psychology of learning and motivation, Academic Press, pp. 193-225 (1988).
(6) Owsley C.: Vision and driving in the elderly, Investigative Ophthalmology \&Visual Science, Vol. 34, No. 11, pp. 31103123 (1993).

(7) Tamida K., Nihei M., Shino M., Kamata M.: An Analysis of the Elderly on Their Driving Behavior Based on the Multimodal Data During the Course Driving, TRANSLOG2007, pp. 369-370 (2007).

(8) Wechsler D: WMS-R Wechsler Memory Scale-Revised Manual (1987).

(9) Sugisita M.: Wechsler Memory Scale - Revised in Japanese, Nihon Bunka Kagakusha (2001)

(10) https://www.google.co.jp/maps/@35.7091605,139.5440584,16z?hl=ja

(11) Rasmussen J.: Human errors - A taxonomy for describing human malfunction in industrial installations, Journal of Occupational Accidents, Vol. 4, No. 2-4, pp. 311-333 (1982).

(12) Kuroda I.: Exploring Human Factors: looking for a way to zero disaster, Anzeneiseishinsyo (1988).

(13) Folstein M., Folstein S., McHugh P.: "Mini-mental state": A practical method for grading the cognitive state of patients for the clinician, Journal of Psychiatric Research, Vol. 12, Issue 3, pp. 189-198 (1975).

(14) Fujita Y., Mimura M., Iijima S.: Correlating driving fitness and functional visual field in the elderly, The Journal of Japanese Occupational Therapy Association, Vol. 31, Issue 3 pp. 233-244 (2012).

(15) Adjutant General's Office: Army Individual Test Battery. Manual of directions and scoring (1944).

(16) Aisen P., Petersen R., Donohue M., Gamst A., Raman R., Thomas R., Walter S., Trojanowski J., Shaw L., Beckett L., Jack C., Jagust W., Toga A., Saykin A., Morris J., Green R., Weiner M.: Alzheimer's Disease Neuroimaging Initiative, Clinical Core of the Alzheimer's Disease Neuroimaging Initiative: progress and plans, Alzheimers Dement, Vol. 6, Issue 3, pp. 239-246 (2010). 